\title{
Mental Health Implications of the COVID-19 Pandemic Among Children and Adolescents: What Do We Know so Far?
}

\author{
Zoe I Listernick ${ }^{1,2}$ \\ Sherif M Badawy (iD ${ }^{3,4}$ \\ 'Ann \& Robert H. Lurie Children's \\ Hospital of Chicago, Chicago, IL, USA; \\ ${ }^{2}$ Department of Medical Education, \\ Northwestern University Feinberg \\ School of Medicine, Chicago, IL, USA; \\ ${ }^{3}$ Division of Hematology, Oncology, and \\ Stem Cell Transplantation, Ann \& Robert \\ H. Lurie Children's Hospital of Chicago, \\ Chicago, IL, USA; ${ }^{4}$ Department of \\ Pediatrics, Northwestern University \\ Feinberg School of Medicine, Chicago, \\ IL, USA
}

\begin{abstract}
The COVID-19 pandemic is an unprecedented time in global history and has many emerging challenges and consequences. While much of the world was focused on the physiological effects and medical interventions or preventions, this article highlights the effects on pediatric mental health. While research is still ongoing, preliminary data suggest a significant impact on the psychosocial wellbeing of the pediatric population. This article hopes to highlight the underlying etiology for this effect and possible mitigations including emphasis on mHealth as well as the future of telemedicine.

Keywords: pediatric, children, adolescents, infants, youth, coronavirus, COVID, COVID-19, pandemic, mental, mental health, psychosocial, psychiatry, psychology, stress, well-being, depression, anxiety, social isolation, social media, racism, digital, mhealth, ehealth, mobile health, interventions, parenting, parents
\end{abstract}

\section{Introduction}

Coronavirus was a novel viral illness that plagued the world in 2019. This virus caused a spectrum of respiratory symptoms, ranging from mild cold-like symptoms to respiratory failure. The medical and public health communities were forced to turn on a dime to address the virus, focusing on research around mitigating the physiological effects through medical interventions and prevention. However, the virus' effect on mental health has not been fully investigated. While there was reportedly a decrease in the number of psychiatric disorders during the COVID-19 pandemic, this is likely due to under- or missed diagnoses. ${ }^{1}$ Furthermore, the effects of the pandemic on pediatric mental health are profound and multifactorial. ${ }^{2}$

Prior to the COVID-19 pandemic, pediatric mental health among children and adolescents has been a growing concern among pediatricians and other health-care providers. Within the United States, $16.5 \%$ of children have at least one mental health disorder, with anxiety and depression being the most common. ${ }^{3}$ Estimated rates of anxiety vary between $5.7 \%$ and $12.8 \%$ for those 18 and younger. ${ }^{4}$ Depression, while less common in pre-pubertal children $(\sim 1-2 \%)$, rises to $4-5 \%$ after puberty and can get as high as $17 \%$ in early adulthood. ${ }^{5}$ Despite the fact that eating disorders (ie, anorexia nervosa and bulimia) are much less common as compared to anxiety and depression, they have been growing in prevalence. The median age of onset is 12 years old and the lifetime prevalence is $0.3 \%$ and $0.9 \%$ for anorexia and bulimia, respectively. According to a 2016 national survey, 49.4\%
Correspondence: Sherif M Badawy Division of Hematology, Oncology, and Stem Cell Transplant, Lurie Ann \& Robert H. Children's Hospital of Chicago, 225

E. Chicago Ave., Chicago, IL, 606I I, USA Tel +l 800-543-7362

Email sbadawy@luriechildrens.org 
of children diagnosed with mental health disorders did not receive the needed treatment. ${ }^{6}$

Screening questionnaires are helpful tools for pediatricians in the community and aid in the diagnosis of mental health disorders. Currently, there are not many recommendations or requirements for screening in pediatric populations. The American Academy of Pediatrics recommends screening all patients greater than 12 years old for depression. This universal screening tool is essential for identifying teenagers at risk for suicidal ideation (SI) and can help caregivers intervene at crucial times. Additionally, there are recommendations to universally screen adolescents for other mental health disorders using the Pediatric Symptom Checklist (PSC-17). If there is a suspicion for other underlying disorders, providers have other screening tools available, such as the Screen for Child Anxiety Related Disorders (SCARED) questionnaire to assess for symptoms of anxiety and the Vanderbilt Questionnaire to screen for Attention-Deficit/Hyperactive Disorder (ADHD). While these tools do exist, it is clear that many children are not getting the help and treatment needed.

\section{Methods}

On June 25, 2021, comprehensive literature search was conducted to identify publications that investigated child and adolescent mental health during the COVID-19 pandemic using PubMed and Google Scholar. Keywords search included: "pediatric mental health," "COVID-19," "Coronavirus," "Anxiety," "Depression." Inclusion criteria for this review were as follows: 1) participants of children or adolescents; 2) studies of any quantitative design; 3) published in English language; 4) conducted nationally or internationally.

\section{Pediatric Mental Health During COVID-19 Pandemic}

The COVID-19 pandemic has had many implications for pediatric populations including school closing, decreased socialization, family deaths, etc. The consequences on mental health are incredibly important to explore in order to adequately treat and care for this patient population. While there are not many large studies on the effect of the pandemic on mental health outcomes, there are many investigators attempting to identify implications and possible solutions.

Many studies have shown that there is a worsening of underlying conditions, such as anxiety and depression, among children and adolescents. One survey distributed by a mental health charity, YoungMinds, assessed 2111 participant who were less than 25 years old with diagnosed psychiatric conditions. Their survey showed that $83 \%$ of participants said that they experienced a worsening of their condition during the pandemic and 26\% responded that they were not able to access the support services needed. ${ }^{7}$ Another survey questioned parents about their children's mental health and $14 \%$ of parents reported worsening of underlying disorders during the pandemic. ${ }^{8}$

There has also been research showing both an increase in anxious and depressive symptoms during the times of COVID-19. Research in China showed that prior to the pandemic, rates of pediatric depression were estimated to be $13.2 \%$, however, this rate increased to $22.28 \%$ during the pandemic. ${ }^{9}$ A second study performed in China supported these results and demonstrated through survey use that out of 1771 adolescents, $28.3 \%$ experienced anxious symptoms and $30.8 \%$ experienced depressive symptoms. ${ }^{10}$ Additionally, studies in the UK have shown that there has been an increase in depressive symptoms by $75 \%$ among children in assessments taken before and during lockdown. ${ }^{11}$ Symptoms of anxiety also increased during quarantine. Self-reported data from Germany showed that rates of anxiety symptoms increased from $15 \%$ to $24 \%$ during COVID-19 using SCARED surveys. ${ }^{12}$ Even following school re-openings, adolescents continued to experience symptoms of anxiety and depression, particularly those with friends of families who were medically or financially impacted by COVID-19. ${ }^{13}$ Rates of eating disorders among children were also affected by the COVID19 pandemic. One study in Australia observed that there was a $104 \%$ increase in childhood admissions for anorexia nervosa. $^{14}$

It is clear that overall rates of mental health disorders and symptoms among the pediatric population have increased. Another metric of effect is emergency department (ED) visits. During COVID-19, multiple studies have shown that emergency department visits for patients of all ages had decreased. However, within the pediatric population the proportion of concerns relating to mental health have increased showing the increase in burden. Center for Disease Control and Prevention data showed that mental health related visits in 2020 increased by $24 \%$ in ages 5-11 and $31 \%$ in ages 12-17 when compared to 2019 data. $^{15}$ Another US study showed that, while pediatric ED visits decreased, visits for mental health conditions increased from $4 \%$ to $5.7 \%$. ${ }^{16}$ There was also shown to be 
an increase in patients screening positively for suicidality and suicide risk. ${ }^{17}$ These metrics indicate the growing concern and need to address mental health within pediatric populations. There are many factors within the global pandemic that influenced mental health and may have caused this increase in rates within children. This article explores the possible causes of worsening mental health burden among this population (Figure 1).

\section{Social Isolation}

The World Health Organization declared a global pandemic on March 11, 2020. Following that date, many countries mandated a lockdown and quarantine for months at a time. This adjustment drastically affected individuals' daily functioning as well as their interactions with others and the world around them. School closures had a profound social impact on children by halting nearly all interactions with their peers. Not surprisingly, the social isolation of quarantine affected rates of mental health disorders and symptoms. A previous study has shown that social isolation is associated with increased risk for SI and self-harm. ${ }^{18}$ Other studies have also shown that social isolation is associated with increase in depressive symptoms. ${ }^{19,20}$ Lack of socialization and confinement to one's home clearly affected the mental health of young individuals.

\section{Family Stress and Parental Mental Health}

After the institution of nation-wide lock downs, families were forced into their homes and were expected to spend more time with their parents, children, and siblings. While this may have strengthened some bonds, this could also lead to a wide array of stressors that impacted children and adolescents. One survey of adolescents in lockdown indicated that there was an association between increased depressive symptoms and lower life satisfaction. ${ }^{21}$ Quarantine also affected parents drastically, who, in turn affect their children's mental health. There were many reasons for increased parental stress, including job loss, the death of family members, or new demands of having to balance at-home work with home-schooling children. There is an association between elevated parental stress and worsening of pediatric mental health. ${ }^{22}$ Children of parents who experienced job loss were also found to be five times more likely to be psychologically mistreated, which includes verbal threatening, belittling, and ridiculing of children. Those who are maltreated exhibit higher rates of aggression, hyperactivity, conduct problems, anxiety, and depression. ${ }^{23}$ In addition, there was an unfortunate global rise in domestic violence during COVID-19 and particularly during lockdown. ${ }^{24}$ Domestic violence is a known Adverse Childhood Event (ACE) and significantly contributes to future physical and mental health of children.

As mentioned previously, there were many stressors for parents during the COVID-19 pandemic, which contributed to a worsening of parental mental health. Parents rated their stress burden and mental health as significantly worse during COVID-19 and reported high rates of anxiety and

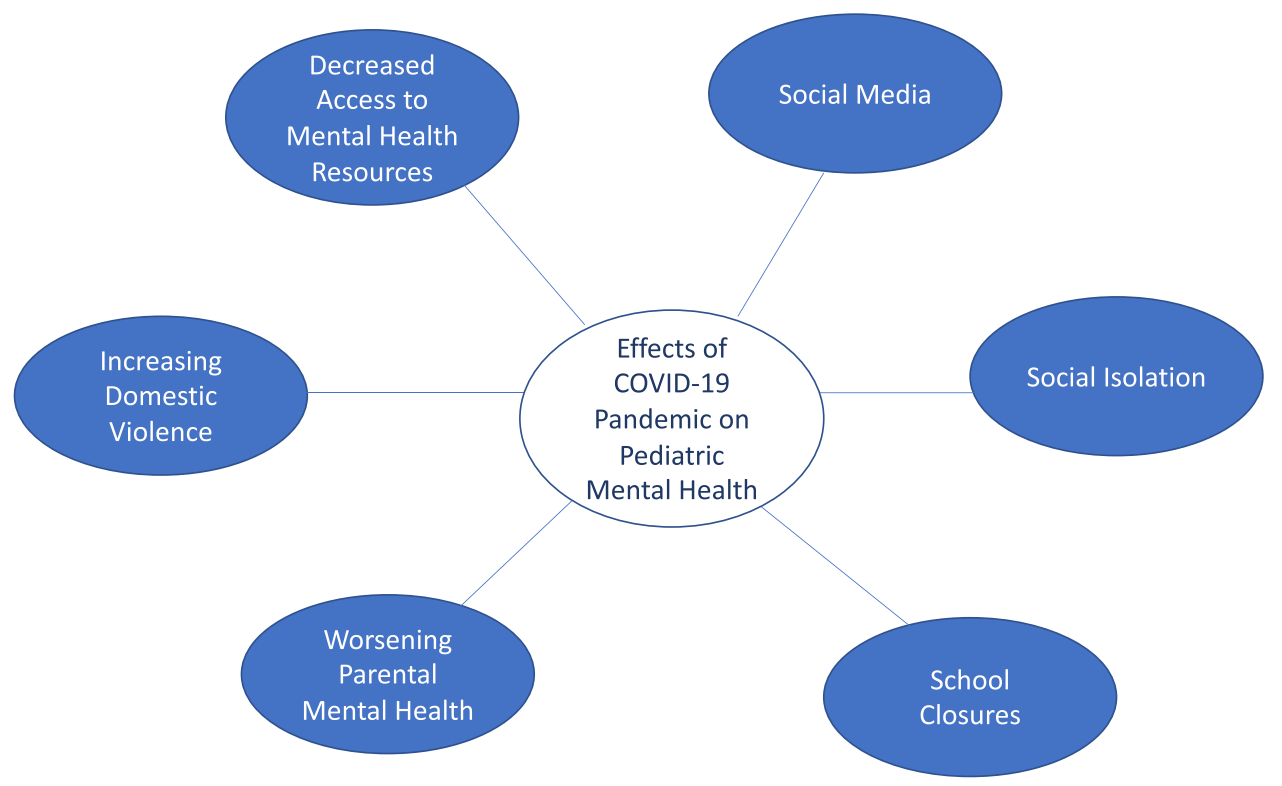

Figure I Mapping of potential mental health implications of the coronavirus (COVID-19) pandemic among children and adolescents. 
depression. ${ }^{25}$ Surveys distributed to parents in Australia showed that, as compared to pre-pandemic data, adults had higher rates of mental health symptoms as well as increased irritability with regards to parenting. ${ }^{26}$ Further survey data showed that parents also had a significant increase in negative mood symptoms. ${ }^{27}$ One study demonstrated that parents who did report higher levels of stress during the pandemic, also reported more childhood ACEs (particularly domestic violence and emotional abuse) ${ }^{25} \mathrm{In}$ addition to an increase in ACEs among households, families with COVID-19-related hardships (ie, income loss and caregiver burden) was associated with an increase in both parental and child negative mood as well as worse sleep. ${ }^{27}$ The increase in parental stress is a clear contributor to worse mental health outcomes in children.

\section{Social Media}

During 2020, there was a drastic increase in the use of new social media platforms. Platforms like TikTok rose to fame and became a staple in the daily life of many children and teenagers. Screen time became essential in an adolescent's life during the pandemic, not only as a source of online education, but as a way to continuously access social media. Data show that $48 \%$ of adolescents spent 5 hours per day on social media and $12 \%$ spent more than 10 hours. ${ }^{28}$ It was found that increases in virtual time was associated with more depressive symptoms. However, it did show that adolescents who spent more time on social media had lower loneliness ratings. ${ }^{28}$ A systematic review showed that social media use was associated with worsening of mental health and psychological distress. ${ }^{29}$

\section{Access to Resources}

Mental health and medical resources were scarce during the beginning of the pandemic and many providers resorted to using virtual medicine. While there are many positives to the implementation of telemedicine, it also comes with many challenges. Children who had previously made personal connections with their therapists, psychiatrists, or psychologists could no longer have a personal, intimate sessions. And while this may have been a good resource and accessible to some, it left many children untreated. There are many households that may not have basic internet access, let alone personal tablets or computers for each individual. Lack of access to technology during the pandemic may lead to untreated mental health disorders and, therefore, worsening symptoms, contributing to increased ED visits and increased suicidality. In addition to children who may not have been pursuing their long-term care, many others may also have delayed seeking initial diagnosis and treatment due to the effects of COVID-19.

\section{Effects of Racism in America During the Pandemic}

The COVID-19 pandemic was an unprecedented time in the United States for multiple reasons. In addition to the global pandemic, the United States was addressing another pandemic of deep-rooted systemic racism. Police killings of unarmed Black men plagued the nation and movements like Black Lives Matter rose to prominence. There were protests on the streets and conversations were happening among families across the country on how to address racism and prejudice. However, as our society began to address these issues in hopes to foster a safer, more inclusive environment, Black youth were also being affected by the ongoing killings. Studies have shown that the increase in police killings of unarmed Black Americans was associated with worse mental health outcomes in the Black community. ${ }^{30}$ Additionally, there was an increase in both depressive and anxious symptoms among Black Americans who had greater self-reported racist encounters. ${ }^{31}$ Unfortunately, the pandemic closed many of the resources that Black youth would seek out for spiritual and mental health services, including local churches. The closure of these churches during the pandemic likely had negative effects on their mental health. ${ }^{32}$

In addition, to the rise in police killings of Black people, there was an increase in xenophobia and racism toward Chinese and Asian Americans. Like Black communities, studies have shown that Asian Americans who perceived higher levels of racism, also experience worse mental health outcomes. ${ }^{31,33}$ The growing concerning and negative experiences among racial minorities was clearly a contributing factor to worsening of mental health during COVID-19.

\section{Implications and Interventions}

It is clear that there are worse mental health outcomes for pediatric populations during COVID-19 compared to prior. The underlying causes are multifactorial, and therefore, there is not a single solution. ${ }^{2}$ However, there are many possible interventions that could help ameliorate the negative impacts of COVID-19 on pediatric mental health, including digital or mobile health (mHealth) behavioral 
interventions with increasing acceptability and promising efficacy. One study has already shown that within the first 4 months of the pandemic, over 100 COVID-related mHealth applications have been created and are available for download. ${ }^{34}$

mHealth has been shown to be a promising intervention for adolescents, particularly in regards to medication adherence. Studies have not only examined how adolescents have a strong interest in adherence-specific apps, ${ }^{35-37}$ but some studies have demonstrated efficacy in increasing medical adherence $^{38}$ as well as targeting mental health outcomes such as emotional distress. ${ }^{39}$ While many these studies areas specific to children with hematologic or oncologic disorders, the findings are suggestive that apps and/or texting may be helpful with medication adherence in other pediatric disorders (ie, psychiatric disease) and therefore preventative of disease progression. One systemic review highlighted that the use of texting or mobile app interventions may be helpful in encouraging preventative behaviors (ie, contraceptive use, physical activity, sexual health, etc). ${ }^{40}$ Additionally, multiple studies in Vietnam demonstrated that while the usage of mHealth apps were low amongst youth, the majority of those who did utilize the apps reported usefulness and satisfaction. ${ }^{41,42}$ This research is very encouraging, particularly when applied to high-risk behavior in mental health disorders. There is room for continued growth and research into the possible effects of mHealth in pediatric populations.

Telehealth and technology play a very promising role in the future of pediatric medicine, particularly mental health. ${ }^{43,44}$ In 2019 , only $8 \%$ of Americans were using telemedicine, however, numbers have increased significantly since the beginning of the pandemic. ${ }^{45}$ To highlight this increase, one study showed that at a single academic center, telemedicine visits increased from 100 per day to over 2200 per day over the course of 1 week during the pandemic. ${ }^{46}$ While physicians and providers are still becoming accustomed to this new type of service, many studies have shown that telehealth is effective in treating mental health disorders. ${ }^{47,48}$ There are many different ways in which technology can be integrated into medicine. Internet-based cognitive behavioral therapy (iCBT) is a telemedicine alternative to in-person CBT that has been recently studied and found to be effective in patients with both anxiety and depression. ${ }^{49}$ More importantly, it has shown to be equivalent to in-person CBT treatment. ${ }^{50}$ This form of digital treatment has also been shown to be effective within other areas of mental health and wellness. For instance, iCBT has shown to be effective in treatment of insomnia $^{51}$ and virtual reality-based CBT has proven to be even more effective than conventional CBT in treatment of binge-purging eating disorders. ${ }^{52} \mathrm{iCBT}$ is a promising telehealth treatment that could be especially beneficial in underserved communities that experience difficulty finding or accessing in-person resources. However, as previously discussed, one of the barriers to this intervention is making it accessible to lower socioeconomic communities who may not have access to internet or the technology to participate in telemedicine.

Another important approach to addressing the burden of worsened mental health is addressing the barriers to socialization. Many of the factors contributing to worse outcomes stem from isolation, which may lead to loneliness or increased stress in the home. However, much of our world is now vaccinated against coronavirus. The CDC currently recommends that all individuals, 12 years and older, receive the vaccine. It is our role as pediatricians to aid in the implementation and distribution of vaccinations to children and adolescents. This will help children safely reintegrate into the opening world and, in turn, improve mental health outcomes.

\section{Conclusions}

The COVID era is an unprecedented time in history and there have been many emerging challenges and consequences of the coronavirus pandemic. While research is still ongoing, preliminary data suggests a significant impact on the psychosocial wellbeing of the pediatric population. This article hopes to highlight the underlying etiology for this effect and possible mitigations. Screening and early recognition of mental health conditions is essential. It is our best interest to utilize technology in order to not only access a wider patient population but to target mental health treatment among children.

\section{Funding}

This project was supported by a grant (K23HL150232, PI: Badawy) from the National Heart, Lung, and Blood Institute of the National Institutes of Health. The content is solely the responsibility of the authors and does not represent the National Institutes of Health.

\section{Disclosure}

The authors report no conflicts of interest in this work. 


\section{References}

1. Williams R, Jenkins DA, Ashcroft DM, et al. Diagnosis of physical and mental health conditions in primary care during the COVID-19 pandemic: a retrospective cohort study. Lancet Public Health. 2020;5 (10):e543-e550. doi:10.1016/S2468-2667(20)30201

2. Serlachius A, Badawy SM, Thabrew H. Psychosocial challenges and opportunities for youth with chronic health conditions during the COVID-19 pandemic. JMIR Pediatr Parent. 2020;3(2):e23057. doi:10.2196/23057

3. Swanson SA, Crow SJ, Le Grange D, Swendsen J, Merikangas KR. Prevalence and correlates of eating disorders in adolescents: results from the national Comorbidity survey replication adolescent supplement. Arch Gen Psychiatry. 2011;68(7):714-723. doi:10.1001/ archgenpsychiatry.2011.22

4. Ramsawh HJ, Chavira DA, Stein MB. Burden of anxiety disorders in pediatric medical settings: prevalence, phenomenology, and a research agenda. Arch Pediatr Adolesc Med. 2010;164 (10):965-972. doi:10.1001/archpediatrics.2010.170

5. Maughan B, Collishaw S, Stringaris A. Depression in childhood and adolescence. J Can Acad Child Adolesc Psychiatry. 2013;22 (1):35-40.

6. Whitney DG, Peterson MD. US national and state-level Prevalence of mental health disorders and disparities of mental health care use in children. JAMA Pediatr. 2019;173(4):389-391. doi:10.1001/ jamapediatrics.2018.5399

7. Lee J. Mental health effects of school closures during COVID-19 [published correction appears in Lancet Child Adolesc Health. 2020 Apr 17]. Lancet Child Adolesc Health. 2020;4(6):421. doi:10.1016/ S2352-4642(20)30109-7

8. Patrick SW, Henkhaus LE, Zickafoose JS, et al. Well-being of parents and children during the COVID-19 pandemic: a national survey. Pediatrics. 2020;146(4):e2020016824. doi:10.1542/peds.2020-016824

9. Duan L, Shao X, Wang Y, et al. An investigation of mental health status of children and adolescents in China during the outbreak of COVID-19. J Affect Disord. 2020;275:112-118. doi:10.1016/j. jad.2020.06.029

10. Ren Z, Xin Y, Wang Z, Liu D, Ho RCM, Ho CSH. What factors are most closely associated with mood disorders in adolescents during the COVID-19 pandemic? A cross-sectional study based on 1771 adolescents in Shandong Province, China. Front Psychiatry. 2021;12:728278. doi:10.3389/fpsyt.2021.728278

11. Bignardi G, Dalmaijer ES, Anwyl-Irvine AL, et al. Longitudinal increases in childhood depression symptoms during the COVID-19 lockdown [published online ahead of print, 2020 Dec 9]. Arch Dis Child. 2020;106(8):791-797. doi:10.1136/archdischild-2020-320372

12. Ravens-Sieberer U, Kaman A, Otto C, et al. Mental health and quality of life in children and adolescents during the COVID-19 pandemic-results of the copsy study. Dtsch Arztebl Int. 2020;117 (48):828-829. doi:10.3238/arztebl.2020.0828

13. Ren Z, Xin Y, Ge J, et al. Psychological impact of COVID-19 on college students after school reopening: a cross-Sectional study based on machine learning. Front Psychol. 2021;12:641806. doi:10.3389/ fpsyg.2021.641806

14. Haripersad YV, Kannegiesser-Bailey M, Morton K, et al. Outbreak of anorexia nervosa admissions during the COVID-19 pandemic. Arch Dis Child. 2021;106(3):e15. doi:10.1136/archdischild-2020-319868

15. Leeb RT, Bitsko RH, Radhakrishnan L, Martinez P, Njai R, Holland KM. Mental health-related emergency department visits among children aged $<18$ years during the COVID-19 pandemic United States, January 1-October 17, 2020. MMWR Morb Mortal Wkly Rep. 2020;69(45):1675-1680. doi:10.15585/mmwr.mm6945a3

16. Krass P, Dalton E, Doupnik SK, Esposito J. US pediatric emergency department visits for mental health conditions during the COVID-19 pandemic. JAMA Netw Open. 2021;4(4):e218533. doi:10.1001/ jamanetworkopen.2021.8533
17. Hill RM, Rufino K, Kurian S, Saxena J, Saxena K, Williams L. Suicide ideation and attempts in a pediatric emergency department before and during COVID-19. Pediatrics. 2021;147(3):e2020029280. doi: $10.1542 /$ peds. $2020-029280$

18. Endo K, Ando S, Shimodera S, et al. Preference for solitude, social isolation, suicidal ideation, and self-harm in adolescents. J Adolesc Health. 2017;61(2):187-191. doi:10.1016/j.jadohealth.2017.02.018

19. Lee CM, Cadigan JM, Rhew IC. Increases in loneliness among young adults during the COVID-19 pandemic and association with increases in mental health problems. J Adolesc Health. 2020;67(5):714-717. doi:10.1016/j.jadohealth.2020.08.009

20. Loades ME, Chatburn E, Higson-Sweeney N, et al. Rapid systematic review: the impact of social isolation and loneliness on the mental health of children and adolescents in the context of COVID-19. J Am Acad Child Adolesc Psychiatry. 2020;59(11):1218-1239.e3. doi:10.1016/j.jaac.2020.05.009

21. Magson NR, Freeman JYA, Rapee RM, Richardson CE, Oar EL, Fardouly J. Risk and protective factors for prospective changes in adolescent mental health during the COVID-19 pandemic. $J$ Youth Adolesc. 2021;50(1):44-57. doi:10.1007/s10964-020-01332-9

22. Whittle S, Bray KO, Lin S, Schwartz O. Parenting and child and adolescent mental health during the COVID-19 pandemic. PsyArXiv. 2020. doi:10.31234/osf.io/ag2r7

23. Lawson M, Piel MH, Simon M. Child maltreatment during the COVID-19 pandemic: consequences of parental job loss on psychological and physical abuse towards children. Child Abuse Negl. 2020;110(Pt 2):104709. doi:10.1016/j.chiabu.2020.104709

24. Boserup B, McKenney M, Elkbuli A. Alarming trends in US domestic violence during the COVID-19 pandemic. Am J Emerg Med. 2020;38(12):2753-2755. doi:10.1016/j.ajem.2020.04.077

25. Calvano C, Engelke L, Di Bella J, et al. Families in the COVID-19 pandemic: parental stress, parent mental health and the occurrence of adverse childhood experiences - results of a representative survey in Germany. Eur Child Adolesc Psychiatry. 2021:1-13. doi:10.1007/ s00787-021-01739-0

26. Westrupp E, Bennett C, Berkowitz TS, et al. Child, parent, and family mental health and functioning in Australia during COVID-19: comparison to pre-pandemic data. Eur Child Adolesc Psychiatry. 2021. doi: $10.31234 /$ osf.io/ydrm 9

27. Gassman-Pines A, Ananat EO, Fitz-Henley J. COVID-19 and parent-child psychological well-being. Pediatrics. 2020;146(4): e2020007294. doi:10.1542/peds.2020-007294

28. Ellis WE, Dumas TM, Forbes LM. Physically isolated but socially connected: psychological adjustment and stress among adolescents during the initial COVID-19 crisis. Can J Behav Sci. 2020;52 (3):177-187. doi:10.1037/cbs0000215

29. Keles B, McCrae N, Grealish A. A systematic review: the influence of social media on depression, anxiety and psychological distress in adolescents. Int J Adolesc Youth. 2020;25(1):79-93. doi:10.1080/ 02673843.2019.1590851

30. Bor J, Venkataramani AS, Williams DR, Tsai AC. Police killings and their spillover effects on the mental health of black Americans: a population-based, quasi-experimental study. Lancet. 2018;392 (10144):302-310. doi:10.1016/S0140-6736(18)31130-9

31. Chae DH, Yip T, Martz CD, et al. Vicarious racism and vigilance during the COVID-19 pandemic: mental health implications among Asian and Black Americans. Public Health Rep. 2021;136 (4):508-517. doi:10.1177/00333549211018675

32. DeSouza F, Parker CB, Spearman-McCarthy EV, et al. Coping with racism: a perspective of COVID-19 church closures on the mental health of African Americans. J Racial and Ethnic Health Disparities. 2021;8(1):7-11. doi:10.1007/s40615-020-00887-4

33. Cheah CSL, Wang C, Ren H, Zong X, Cho HS, Xue X. COVID-19 racism and mental health in Chinese American Families. Pediatrics. 2020;146(5):e2020021816. doi:10.1542/peds.2020-021816 
34. Zhang MWB, Chow A, Ho RCM, Smith HE. An overview of commercially available apps in the initial months of the COVID-19 pandemic. Front Psychiatry. 2021;12:557299. doi:10.3389/ fpsyt.2021.557299

35. Heneghan MB, Hussain T, Barrera L, et al. Access to technology and preferences for an mHealth intervention to promote medication adherence in pediatric acute lymphoblastic leukemia: approach leveraging behavior change techniques. J Med Internet Res. 2021;23(2): e24893. doi: $10.2196 / 24893$

36. Alberts NM, Badawy SM, Hodges J, et al. Development of the InCharge health mobile app to improve Adherence to hydroxyurea in patients with sickle cell disease: user-centered design approach. JMIR Mhealth Uhealth. 2020;8(5):e14884. doi:10.2196/14884

37. Badawy SM, Thompson AA, Liem RI. Technology access and smartphone app preferences for medication adherence in adolescents and young adults with sickle cell disease. Pediatr Blood Cancer. 2016;63 (5):848-852. doi:10.1002/pbc.25905

38. Badawy SM, Barrera L, Sinno MG, Kaviany S, O'Dwyer LC, Kuhns LM. Text messaging and mobile phone apps as interventions to improve adherence in adolescents with chronic health conditions: a systematic review. JMIR Mhealth Uhealth. 2017;5(5):e66. doi:10.2196/mhealth.7798

39. Ramsey WA, Heidelberg RE, Gilbert AM, Heneghan MB, Badawy SM, Alberts NM. eHealth and mHealth interventions in pediatric cancer: a systematic review of interventions across the cancer continuum. Psychooncology. 2020;29(1):17-37. doi:10.1002/ pon. 5280

40. Badawy SM, Kuhns LM. Texting and mobile phone app interventions for improving adherence to preventive behavior in adolescents: a systematic review. JMIR Mhealth Uhealth. 2017;5(4):e50. doi: 10.2196/mhealth.6837

41. Do TTT, Le MD, Van Nguyen T, et al. Receptiveness and preferences of health-related smartphone applications among Vietnamese youth and young adults. BMC Public Health. 2018;18(1):764. doi:10.1186/ s12889-018-5641-0

42. Tran BX, Zhang MW, Le HT, et al. What drives young Vietnamese to use mobile health innovations? Implications for health communication and behavioral interventions. JMIR Mhealth Uhealth. 2018;6 (11):e194. doi:10.2196/mhealth.6490
43. Shah AC, Badawy SM. Telemedicine in pediatrics: systematic review of randomized controlled trials. JMIR Pediatr Parent. 2021;4(1): e22696. doi:10.2196/22696

44. Shah AC, O'Dwyer LC, Badawy SM. Telemedicine in malignant and nonmalignant hematology: systematic review of pediatric and adult studies. JMIR Mhealth Uhealth. 2021;9(7):e29619. doi:10.2196/29619

45. Badawy SM, Radovic A. Digital approaches to remote pediatric health care delivery during the COVID-19 pandemic: existing evidence and a call for further research. JMIR Pediatr Parent. 2020;3 (1):e20049. doi:10.2196/20049

46. Contreras CM, Metzger GA, Beane JD, Dedhia PH, Ejaz A, Pawlik TM. Telemedicine: patient-provider clinical engagement during the COVID-19 pandemic and beyond. $J$ Gastrointest Surg. 2020;24(7):1692-1697. doi:10.1007/s11605-020-04623-5

47. Hilty DM, Ferrer DC, Parish MB, Johnston B, Callahan EJ, Yellowlees PM. The effectiveness of telemental health: a 2013 review. Telemed J E Health. 2013;19(6):444-454. doi:10.1089/ tmj.2013.0075

48. Bashshur RL, Shannon GW, Bashshur N, Yellowlees PM. The empirical evidence for telemedicine interventions in mental disorders. Telemed J E Health. 2016;22(2):87-113. doi:10.1089/ tmj.2015.0206

49. Grist R, Croker A, Denne M, Stallard P. Technology delivered interventions for depression and anxiety in children and adolescents: a systematic review and meta-analysis. Clin Child Fam Psychol Rev. 2019;22(2):147-171. doi:10.1007/s10567-018-0 271-8

50. Carlbring P, Andersson G, Cuijpers P, Riper H, Hedman-Lagerlöf E. Internet-based vs. face-to-face cognitive behavior therapy for psychiatric and somatic disorders: an updated systematic review and meta-analysis. Cogn Behav Ther. 2018;47(1):1-18. doi:10.1080/ 16506073.2017.1401115

51. Soh HL, Ho RC, Ho CS, Tam WW. Efficacy of digital cognitive behavioural therapy for insomnia: a meta-analysis of randomised controlled trials. Sleep Med. 2020;75:315-325. doi:10.1016/j. sleep.2020.08.020

52. Low TL, Ho R, Ho C, Tam W. The efficacy of virtual reality in the treatment of binge-purging eating disorders: a meta-analysis. Eur Eat Disord Rev. 2021;29(1):52-59. doi:10.1002/erv.2804
Pediatric Health, Medicine and Therapeutics

\section{Publish your work in this journal}

Pediatric Health, Medicine and Therapeutics is an international, peerreviewed, open access journal publishing original research, reports, editorials, reviews and commentaries. All aspects of health maintenance, preventative measures and disease treatment interventions are addressed within the journal. Practitioners from all disciplines are invited to submit their work as well as healthcare researchers and patient support groups. The manuscript management system is completely online and includes a very quick and fair peer-review system. Visit http://www.dovepress.com/testimonials.php to read real quotes from published authors. 